\title{
Unbiased PCR-free spatio-temporal mapping of the mtDNA mutation spectrum reveals brain region-specific responses to replication instability
}

Emilie Kristine Bagge ${ }^{1}$, Noriko Fujimori-Tonou ${ }^{1,2}$, Mie Kubota-Sakashita ${ }^{1}$, Takaoki Kasahara ${ }^{1,3}$ and Tadafumi Kato ${ }^{1,4^{*}}$ (D)

\begin{abstract}
Background: The accumulation of mtDNA mutations in different tissues from various mouse models has been widely studied especially in the context of mtDNA mutation-driven ageing but has been confounded by the inherent limitations of the most widely used approaches. By implementing a method to sequence mtDNA without PCR amplification prior to library preparation, we map the full unbiased mtDNA mutation spectrum across six distinct brain regions from mice.

Results: We demonstrate that ageing-induced levels of mtDNA mutations (single nucleotide variants and deletions) reach stable levels at 50 weeks of age but can be further elevated specifically in the cortex, nucleus accumbens (NAc), and paraventricular thalamic nucleus (PVT) by expression of a proof-reading-deficient mitochondrial DNA polymerase, Polg ${ }^{\text {D181A }}$. The increase in single nucleotide variants increases the fraction of shared SNVs as well as their frequency, while characteristics of deletions remain largely unaffected. In addition, Polg ${ }^{D 181 A}$ also induces an ageing-dependent accumulation of non-coding control-region multimers in NAc and PVT, a feature that appears almost non-existent in wild-type mice.

Conclusions: Our data provide a novel view of the spatio-temporal accumulation of mtDNA mutations using very limited tissue input. The differential response of brain regions to a state of replication instability provides insight into a possible heterogenic mitochondrial landscape across the brain that may be involved in the ageing phenotype and mitochondriaassociated disorders.
\end{abstract}

Keywords: Mitochondrial DNA, Polymerase gamma, Polg, Ageing, Mitochondrion, mtDNA mutation, Paraventricular thalamic nucleus, Nucleus accumbens, Substantia nigra, Dorsal raphe

\section{Background}

Ageing is characterised by diverse molecular, physiological, and behavioural changes, the mechanistic onset of which is poorly understood. Time-dependent

\footnotetext{
* Correspondence: tadafumi.kato@juntendo.ac.jp

'Laboratory for Molecular Dynamics of Mental Disorders, RIKEN Center for Brain Science, Wako, Saitama, Japan

${ }^{4}$ Department of Psychiatry and Behavioral Science, Juntendo University, Graduate School of Medicine, Hongo 2-1-1, Bunkyo, Tokyo 113-8421, Japan Full list of author information is available at the end of the article
}

accumulation of mitochondrial DNA (mtDNA) mutations through a vicious cycle of reactive oxygen species (ROS) production and oxidative damage to mtDNA has been proposed as a possible driver [1], causing ageinginduced mitochondrial dysfunction. However, accumulating evidence suggests that mtDNA is not associated with oxidative damage $[2,3]$, despite the presence of other oxidative damage in mitochondria $[4,5]$. Though it has been highly debated whether mtDNA mutations

C The Author(s). 2020 Open Access This article is licensed under a Creative Commons Attribution 4.0 International License, which permits use, sharing, adaptation, distribution and reproduction in any medium or format, as long as you give appropriate credit to the original author(s) and the source, provide a link to the Creative Commons licence, and indicate if changes were made. The images or other third party material in this article are included in the article's Creative Commons licence, unless indicated otherwise in a credit line to the material. If material is not included in the article's Creative Commons licence and your intended use is not permitted by statutory regulation or exceeds the permitted use, you will need to obtain permission directly from the copyright holder. To view a copy of this licence, visit http://creativecommons.org/licenses/by/4.0/ The Creative Commons Public Domain Dedication waiver (http://creativecommons.org/publicdomain/zero/1.0/) applies to the data made available in this article, unless otherwise stated in a credit line to the data. 
are cause or effect of ageing [3], mtDNA mutations alone are adequate to drive ageing as seen in the mutator mouse, an ageing model expressing a proof-readingdeficient mitochondrial DNA polymerase Polg, Polg ${ }^{D 257 A}$ [6]. Evidence suggests the involvement of specific mtDNA mutations in oxidative stress [7,8], indicating that the 37 genes encoded by mtDNA (13 proteins, 22 tRNAs, and 2 rRNAs) as well as the non-coding control region (NCR) have different contributions to mitochondrial dysfunction. As the NCR interacts with the inner mitochondrial membrane [9] and several proteins [1012] to form the nucleoid structure and regulate transcription and replication, mitochondria may be highly dependent on the NCR for proper function. What remains unclear is the exact spatio-temporal accumulation of mtDNA mutations between individual organs and even more importantly, the heterogeneity with which mtDNA mutations may accumulate across an organ.

We have developed a variation of the mutator mouse that expresses an alternative proof-reading-deficient Polg, Polg ${ }^{D 181 A}$, under the control of the CaMKII $\alpha$-promoter, resulting in forebrain neuron-specific expression of the transgene [13]. This model can be used to examine the neuron-specific mitochondrial response to replication instability arising from lack of proof-reading of Polg, and we have previously demonstrated the accumulation of dysfunctional mitochondria in these mice $[14,15]$.

Up until now, purified mitochondria from whole organs [16] or some form of either partial or nearly fulllength PCR amplification of mtDNA [17-19] have been required for next-generation sequencing. Especially in highly heterogeneous tissue such as the brain, the cellular composition, metabolic profile, and local environment could potentially harbour regional mutational hotspots that may contribute to both the ageing phenotype and various disorders, but such limited tissue regions have been too small to study without PCR amplification. PCR amplification not only introduces a bias to the study of mtDNA, it also makes it difficult to identify rearrangements of mtDNA such as deletions and duplications unless full-length amplification of mtDNA is performed.

In this study, we implemented a method to prepare mtDNA for next-generation sequencing without PCR amplification prior to library preparation using DNA extracted from small brain dissections from mice during ageing. We show that mice accumulate single nucleotide variants (SNVs) and deletions with ageing across all brain regions in a largely homogenous manner. However, the expression of Polg ${ }^{D 181 A}$ causes a highly brain region-specific increase in the ageing-induced accumulation of SNVs and deletions. Our data demonstrate a previously undescribed bimodal distribution of deletion sizes across both genotypes. In addition, we demonstrate a Polg ${ }^{D 181 A}$-dependent and ageing-induced accumulation of NCR-containing multimers. In all, our unbiased approach to map the full spatio-temporal mtDNA mutation spectrum provides an unprecedented method to gain insight into brain-wide mitochondrial heterogeneity.

\section{Results \\ Implementation of a method for isolation of mtDNA from small tissue samples}

To investigate the full mtDNA mutation spectrum in small mouse brain regions and avoid the inherent bias present in PCR amplification, we implemented a method for the enzymatic depletion of nuclear DNA (nDNA) from total DNA [20] extracted from brain tissue. We enzymatically depleted nDNA from total DNA by treatment with exonuclease $\mathrm{V}$, an enzyme that targets the free ends of linear DNA essentially leaving circular mtDNA intact [20]. We used mtDNA-enriched samples directly for library preparation for next-generation sequencing (Fig. 1a).

We allowed for split-read mapping to identify deletions with BBMap [21] (Fig. 1b) using a custom mtDNA reference composed of two mm10 MT references in tandem (dMT). In a two-round mapping approach, we removed residual nDNA-derived sequencing reads, especially due to the presence of nuclear mitochondrial DNA segments (Numts), i.e. mtDNA-like sequences in the nuclear genome. Due to the circularity of mtDNA (Fig. 1c), deletions may span the "ends" of the mtDNA reference, that is linear in nature, which will interfere with deletion calling. By using dMT, we circumvented this and were able to reliably detect variants at any position in mtDNA. As sequencing reads generated by Nextera are well known to exhibit GC bias in the first bases of the read, we trimmed these bases and excluded an additional $5 \mathrm{bp}$ at the read ends during variant calling (see the "Methods" section). Based on this, we have no reason to believe that variant calling is influenced by transposase sequence bias. For identification of mtDNA variants, we sampled the sensory cortex (COR), caudate putamen (CP), dorsal raphe (DR), nucleus accumbens (NAc), paraventricular thalamic nucleus (PVT), and substantia nigra (SN) (Fig. 1d) during mouse ageing and confirmed nDNA depletion by qPCR before library prep and sequencing (Fig. 1e).

\section{Ageing-related accumulation of SNVs and deletions across all brain regions}

We initially mapped the ageing-related changes in mtDNA mutations across the brains of 10-, 50-, and 80week-old wild-type (WT) mice (Fig. 1f). As expected, the number of SNVs in 10-week-old mice was very low but rose on average 10-fold in 50-week-old mice and remained relatively unchanged at 80 weeks (Fig. 1f, left). 


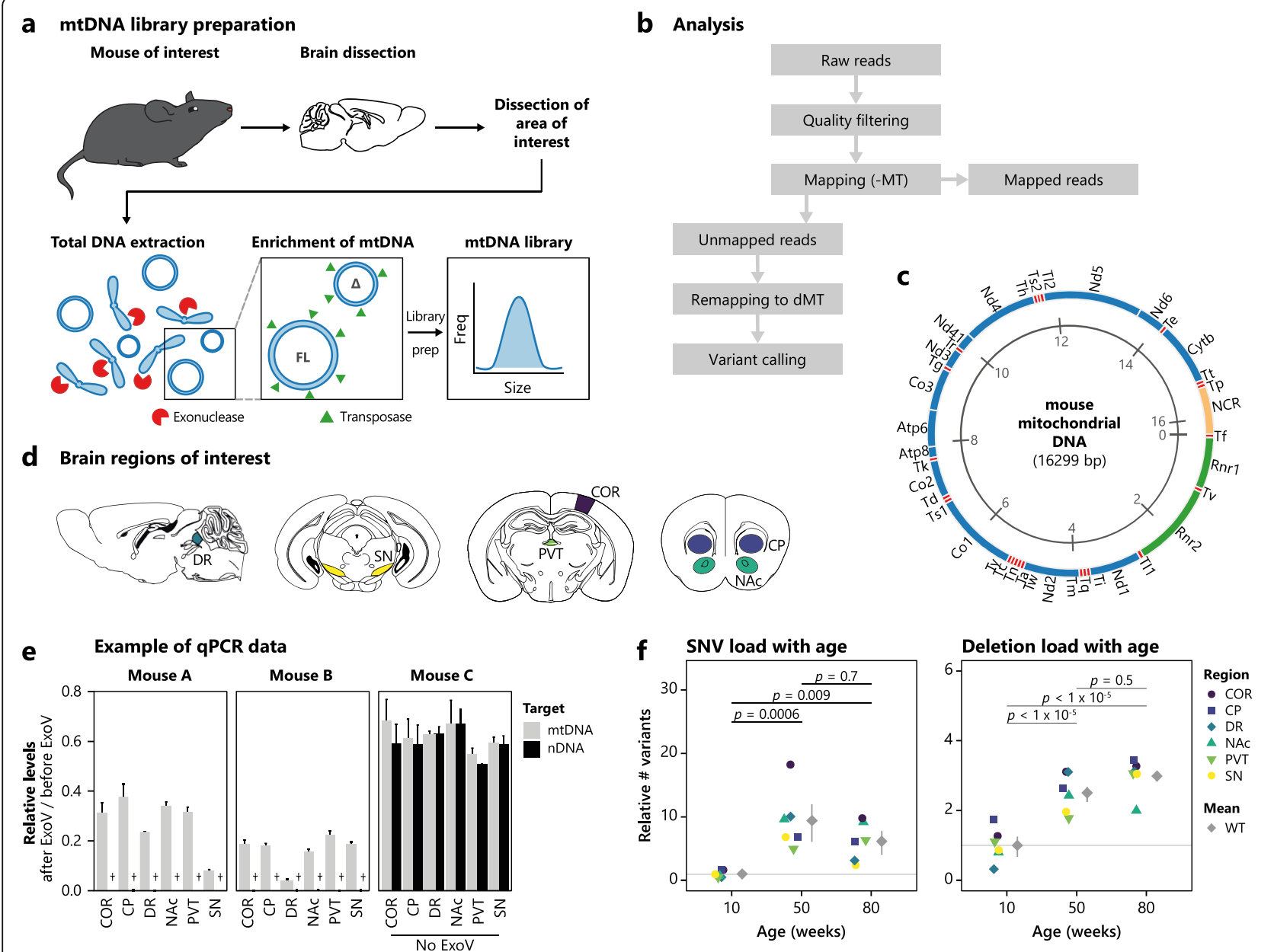

Fig. 1 Ageing increases the load of both SNVs and deletions in mtDNA across all brain regions. a Schematic illustration of the workflow from mouse to prepared library. Briefly, brain regions of interest were rapidly sampled and total DNA was extracted. Linear DNA was enzymatically degraded by exonuclease (ExOV), and non-linear DNA is purified and used for library preparation. FL: full-length mtDNA molecule, $\triangle$ : mtDNA molecule with deletion. $\mathbf{b}$ Overview of the analysis workflow to optimise mtDNA variant detection. Shortly, after quality filtering, reads were mapped to $\mathrm{mm} 10$ without the mitochondrial chromosome (MT). Unmapped reads were then re-mapped to a modified MT reference (dMT: two MT references in tandem) and variants called. c Overview of mouse mtDNA. Green: rRNA encoding genes; blue: protein-coding genes; red: tRNAencoding genes; orange: non-coding region (NCR). d Schematic showing the areas isolated as the cortex (COR), caudate putamen (CP), dorsal raphe (DR), nucleus accumbens (NAc), paraventricular nucleus of the thalamus (PVT), and substantia nigra (SN). e DNA stored before and after ExoV digestion was subjected to GPCR to determine the relative levels of three mtDNA and three nuclear targets before and after digestion (shown for two different mice, A and B). Mouse $\mathrm{C}$ was treated as $\mathrm{A}$ and $\mathrm{B}$ but without the addition of ExoV. Bars show the mean of target signals and the standard deviation is indicated. t: nDNA after ExoV treatment was not detected or only detected at a very low level by qPCR and may not be visible in the bar plot. $\mathbf{f}$ Dot plot illustrating the age-dependent increase in the load of SNVs (left) and deletions (right) across the investigated brain regions (as indicated by the colour legend). All samples have been normalised to the mean of the variants at 10 weeks. Grey diamonds indicate the mean of all regions at the indicated age, and the $95 \%$ confidence interval is shown. Three-way ANOVA showed age, not region or animal, significantly $(p<0.01)$ contributed to SNV and deletion levels. Tukey's test was used post hoc to determine $p$ values between each age group

Similarly, deletions also reached a plateau at 50 weeks after increasing 2.5-3-fold from 10 weeks (Fig. 1f, right). The plateau reached in both SNVs and deletions at 50 weeks indicates a restriction in the load of mtDNA mutations. This may be imposed by loss of mitochondria function, thus limiting its propagation or triggering mitophagy. Alternatively, selective replication of mtDNA molecules may keep the mutation load from further increasing. In all, both SNVs and deletions appeared homogenously accumulated across the examined brain regions during ageing, but may be influenced by different pathological settings.

\section{Polg ${ }^{D 181 A}$ expression causes brain region-specific SNV accumulation with ageing}

Having established that our method could be used to map ageing-induced mtDNA mutations, we turned to 
our Polg ${ }^{D 181 A}$ model mice to investigate the brain region-specific influence of proof-reading deficiency. We sequenced mtDNA from the six brain regions of interest from Polg ${ }^{D 181 A}$ mice and identified the SNVs in each region at 10, 50, and 80 weeks of age (Fig. 2a).

In young mice, there was no change in SNV levels between WT and Polg ${ }^{D 181 A}$ in 10 -week-old mice. At 50 and 80 weeks, we observed a significant increase in SNV levels which was especially prominent in COR, NAc, and PVT (Fig. 2a). This demonstrated that the heterogeneity of the mitochondrial response to proof-reading deficiency is present across an organ and not only between organs $[6,16,22,23]$.

Importantly, we found no relationship between the expression of the Polg ${ }^{D 181 A}$ transgene in the investigated brain regions and the level of detected SNVs (Additional file 1: Fig. S5c).

\section{SNVs are excessively shared between brain regions}

We wondered whether the increase in SNVs with both ageing and Polg ${ }^{D 181 A}$ expression was affecting the same positions in mtDNA across brain regions. Indeed, looking in 10-bp non-overlapping intervals, we found an increase in shared SNV positions with ageing which was enhanced by Polg ${ }^{D 181 A}$ expression (Fig. 2b). The overlap between individual animals was most prominent in PVT and NAc (Fig. 2c). As Polg ${ }^{D 181 A}$ expression introduced a shift of the SNV distribution to the right side of the distribution plot (i.e. towards the NCR) compared to WT (Fig. 2d), we examined where shared SNVs were located.
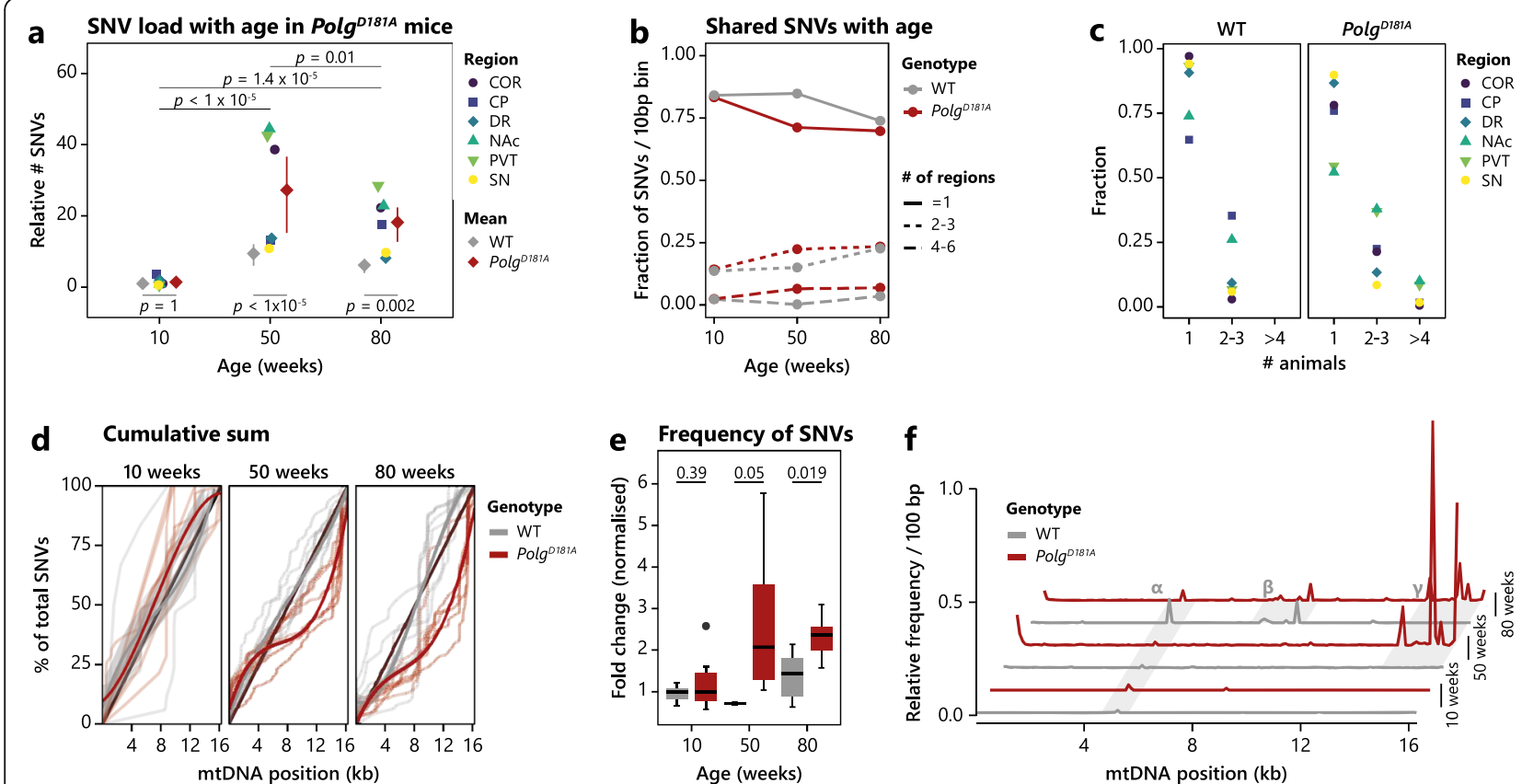

Fig. 2 SNVs heterogeneously accumulate across brain regions in Polg ${ }^{\text {181A }}$ mice and cause mtDNA position-specific mutational patterns. a Dot plot illustrating the age-dependent increase in the load of SNVs in Polg ${ }^{D 181 A}$ mice across the investigated brain regions (as indicated by the colour legend) normalised to the mean of WT samples at 10 weeks. Grey diamonds indicate the mean of WT-derived brain region samples for reference (same as in Fig. 1f). Red diamonds indicate the mean of Polg ${ }^{D 181 A}$-derived brain region samples and the $95 \%$ confidence interval is shown. Three-way ANOVA (age, region, and animal) of Polg D181A-derived samples showed that age significantly contributed to SNV levels ( $p$ values of post hoc Tukey's test are shown). Three-way ANOVA showed a significant contribution of all variables (age, genotype, region). $p$ values of post hoc Tukey's test comparing WT and Polg ${ }^{181 A}$ at each age are shown. For region contribution, we found a significant contribution of COR, NAc, and PVT to SNV levels in Polg ${ }^{\text {181A }}$ mice using a linear model for main effects. b SNVs were counted in 10-bp non-overlapping bins for WT (grey) and Polg ${ }^{D 181 A}$ (red) mice at 10, 50, and 80 weeks, and the number of regions with SNV in each bin calculated. Note that in the case that one region has more than one SNV in a bin, it is only counted as one instance of an SNV. The overlap was visualised for non-overlapping bins $(" 1 ")$, bins shared across two or three regions ("2-3"), and bins shared across four to six regions ("4-6"). c SNVs were counted in 10-bp nonoverlapping bins for WT (grey) and Polg ${ }^{\text {D181A }}$ (red) mice at 50 weeks, and the number of individual animals with SNVs in each bin calculated. Note that in the case that one animal has more than one SNV in a bin, it is only counted as one instance of an SNV. The overlap was visualised for non-overlapping bins $(" 1 ")$, bins shared across two or three animals ("2-3"), and bins shared by four or more animals $(" \geq 4$ "). $\mathbf{d}$ Cumulative percentage of SNVs detected in each examined brain region (thin lines) for both WT (grey) and Polg ${ }^{D 181 A}$ (red) at 10, 50, and 80 weeks old. Bold lines indicate the smooth conditional mean for each genotype. e The relative average SNV allele frequency for each region for WT (grey) and Polg ${ }^{D 181 A}$ (red) mice at 10, 50, and 80 weeks as indicated shown as boxplots. $p$ values of two-sided $t$ tests are shown. f SNVs across brain regions were pooled for each genotype at each age and divided into 100-bp bins across the mtDNA reference and the allele fraction for SNVs in each bin summed and normalised (i.e. highest peak set to 1). Grey areas indicate mtDNA regions where peaks are found across all variables (a), peaks that are ageing-dependent $(\beta)$, and ageing-induced Polg ${ }^{D 181 A}$-dependent peaks $(\gamma)$ 
We found that shared SNV positions were significantly different from non-shared SNV positions in Polg ${ }^{D 181 A}$ mice ( $t$ test, $p<1 \times 10^{-5}$ ) but not in WT ( $t$ test, $p=$ $0.254)$ when looking across all ages and shifted towards the 3 ' region (i.e. towards the NCR) (Additional file 1: Fig. S1a).

The increase in shared SNVs was accompanied by a significant increase in SNV frequency with Polg ${ }^{D 181 A}$ expression (Fig. 2e) which was driven by high frequency SNVs in specific mtDNA regions (Fig. 2f). These regions appeared highly context-dependent, i.e. peaks that are found across all samples (" $\alpha$ " on Fig. $2 f$ ), only in very aged mice (" $\beta$ "), or are Polg $^{D 181 A}$-specific (" $\gamma$ "). This was mimicked in the Pearson correlation, where most brain regions from 50- and 80-week-old Polg ${ }^{D 181 A}$ mice form a distinct cluster and most samples from 10-week-old mice form a distinct cluster (Additional file 1: Fig. S1b) and we found specific SNV hotspots in 10-week-old animals independent of genotype (Additional file 1: Fig. S1c). In addition, there was a significant overlap of the specific positions at which SNVs are present in COR, NAc, and PVT (the brain regions most sensitive to Polg ${ }^{D 181 A}$ expression) at both 50 and 80 weeks in Polg ${ }^{D 181 A}$ mice (Additional file 1: Fig. S1d, right). For WT mice, the overlap is only pronounced at 80 weeks and $p$ values do not reach similar levels of significance (Additional file 1: Fig. S1d, left).
We found an increase of SNVs in the NCR and complex III genes (Additional file 1: Fig. S1e), while transitions and transversions (Additional file 1: Fig. S1f) were comparable to those of previous studies, and we saw no indication of either ageing- or Polg ${ }^{D 181 A}$-induced oxidative mutations [19, 24], together with no change in the types of mutations (Additional file 1: Fig. S1g).

Together, these data demonstrated a brain regionspecific ageing-dependent Polg ${ }^{D 181 A}$-induced mtDNA SNV spectrum, where COR, NAc, and PVT are regional hotspots. In addition, certain mtDNA positions are highly sensitive to SNVs and seem to function as context-dependent mutational hotspots.

\section{Ageing-dependent Polg ${ }^{D 181 A}$-induced deletions accumulate in the same brain regions as SNVs}

We next turned our attention to the influence of Polg ${ }^{D 181 A}$ expression on the accumulation of deletions. We found a significant ageing-induced accumulation of deletions in both 50- and 80- compared to 10-week-old Polg ${ }^{D 181 A}$ mice, but we observed no significant differences between WT and Polg ${ }^{D 181 A}$ at any age (Fig. 3a). However, deletion accumulation in response to Polg ${ }^{D 181 A}$ showed a prominent region specificity. While CP, DR, and SN Polg ${ }^{D 181 A}$ SNV levels were only slightly elevated compared to WT mice, COR, NAc, and PVT showed a very high accumulation of deletions at 50 and

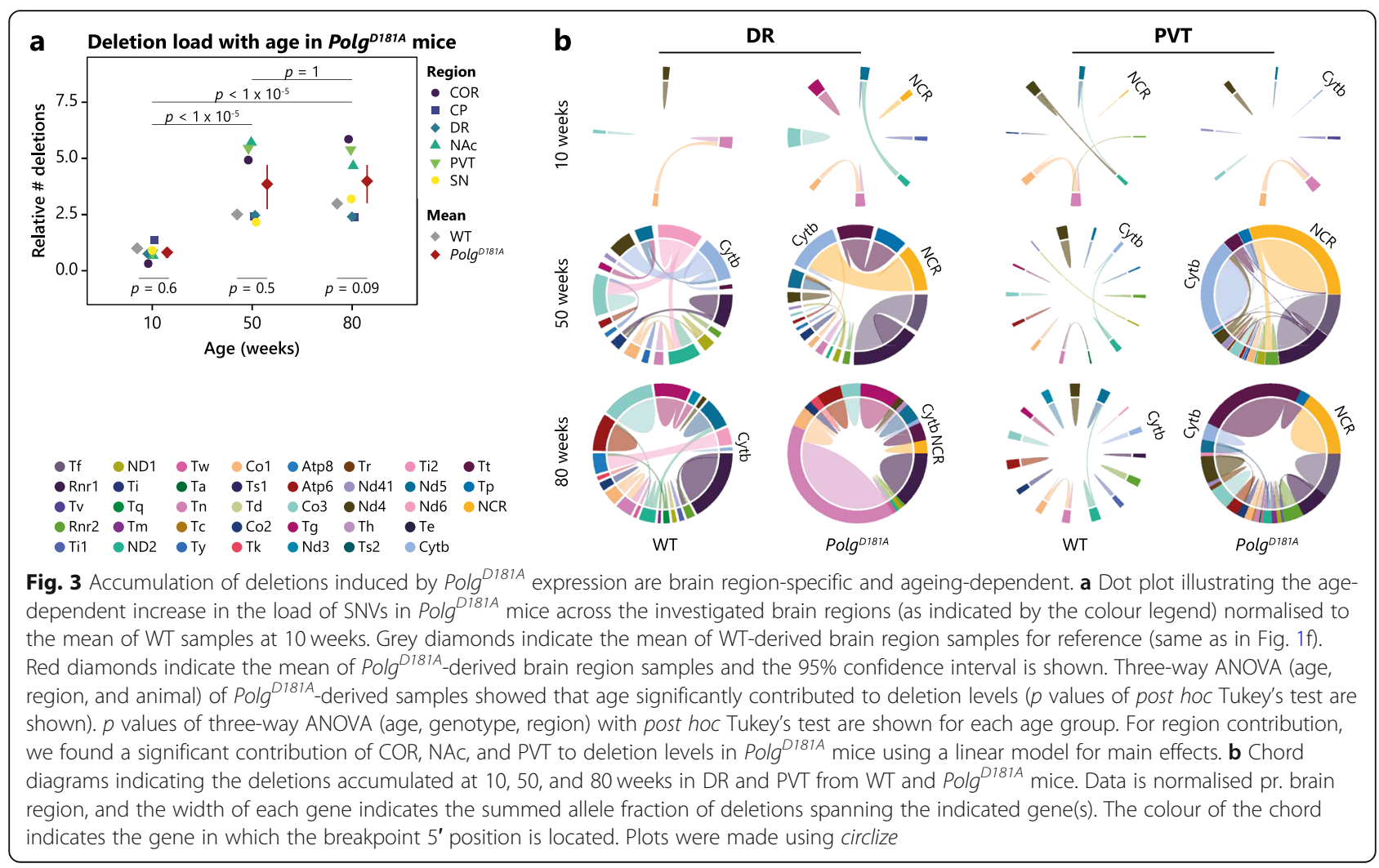


80 weeks. We found a significant difference in deletion levels between these regions compared to the other regions in Polg ${ }^{D 181 A}$ when pooling data from 50- and 80week-old mice ( $p=0.002$, one-way ANOVA). Similar to SNVs, we found no indication that expression levels of the Polg ${ }^{D 181 A}$ transgene were the major driver of deletion levels in the Polg ${ }^{D 181 A}$ mice (Additional file 1: Fig. S5c).

The differences across brain regions with ageing of WT and Polg ${ }^{D 181 A}$ mice can be appreciated by chord diagrams showing the span of all deletions at each time point (Fig. 3b). Where PVT showed both an ageinginduced and a clear ageing-dependent Polg $^{D 181 A}$-induced accumulation of deletions, DR only showed an ageinginduced accumulation of deletions, highlighting the brain region-specific mtDNA sensitivity to a setting of replication instability. Pearson correlation indicated similarities in the deletions found with ageing of Polg ${ }^{D 181 A}$ mice (Additional file 1: Fig. S2a), indicating that Polg ${ }^{D 181 A}$ expression induces a specific landscape of mtDNA deletions.

\section{Deletions share characteristics independent of genotype}

The positions at which deletions start and end are termed breakpoints and based on the co-occurring deletions between brain regions from Polg ${ }^{D 181 A}$ mice, we hypothesised that breakpoints must be shared between different samples. We looked in 100-bp bins along the mtDNA and found that shared breakpoints cluster in very distinct locations (Additional file 1: Fig. S2b). Some shared breakpoints are age- and genotype-independent $(\sim 5 \mathrm{~kb})$ whereas others are genotype-dependent $(\sim 15$ kb). Sizes of deletions themselves follow a bimodal distribution independent of age and genotype and can be roughly divided into those $<100 \mathrm{bp}$ and those $>1 \mathrm{~kb}$, with few observations in the intermediate range (Fig. 4a). Even though the number of deletions in 10-week-old animals is low, they still follow this distribution, though the fraction of very small deletions is high compared to aged animals.

\section{Molecular determinants of deletions}

A previous study has suggested that the majority of mtDNA deletions in Parkinson's patients occur at direct repeats [17], a proposed [25] though highly debated [26] feature of human mtDNA deletions. To investigate the influence of direct repeats in breakpoint formation in the mouse brain, we identified direct repeats $\geq 8 \mathrm{bp}$ in mtDNA (Additional file 1: Fig. S2c). After pooling deletions per genotype, we identified the direct repeat pair with the shortest average distance from the $5^{\prime}$ and $3^{\prime}$ breakpoints of WT and Polg ${ }^{D 181 A}$ deletions as well as for in silico generated, deletion length-matched deletion libraries for each genotype (see the "Methods" section). The shortest average distance was shorter for experimentally derived deletions than randomly generated deletions for both WT and Polg ${ }^{D 181 A}$ mice (Fig. 4b), but there was no difference between WT and Polg ${ }^{D 181 A}(t$ test, $p=0.905)$. This indicates that direct repeats may contribute to at least a part of the identified deletions. We found this to be the case at 10 and 50 weeks but not 80 weeks (Additional file 1: Fig. S2d), as deletions are significantly closer to direct repeats than the in silico deletion libraries for both WT and Polg ${ }^{D 181 A}$ mice.

Restriction of sequence similarity to direct repeats is a rigorous criterion. We therefore calculated the sequence
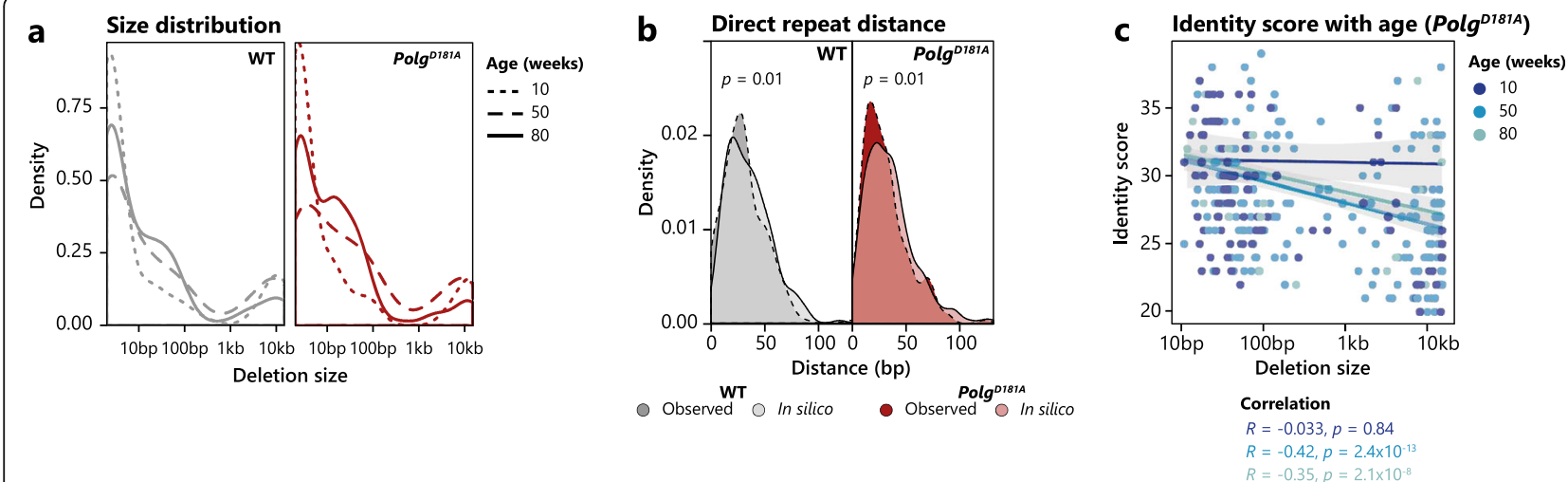

Fig. 4 Characteristics of deletions change with age, but not the expression of Polg ${ }^{D 181 A}$. a Density plot of deletion sizes for WT (grey) and Polg ${ }^{\text {181A }}$ (red) for 10- (dotted line), 50- (dashed line), and 80-week-old (full line) mice. b The shortest average distance from 5' and 3' deletion breakpoint pairs to a direct repeat pair in the mitochondrial genome for the observed deletions (darker colour) and a random in silico generated

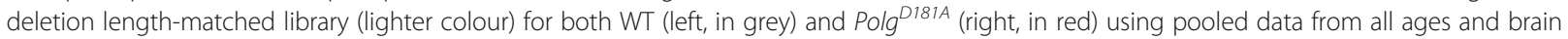
regions examined for each genotype. $p$ values of two-sided $t$ tests are shown. c Needle identity score calculated in a \pm 10 bp window at the $5^{\prime}$ and $3^{\prime}$ deletion breakpoints as a function of deletion size after pooling of WT and Polg ${ }^{D 181 A}$ samples. Correlation for each age is indicated by the full lines and correlation data indicated in the same colour code 
identity scores in a 20-bp window surrounding all $5^{\prime}$ and 3' breakpoints (i.e. $10 \mathrm{bp}$ on each side of the breakpoint). We found a negative correlation between sequence identity score and deletion length in 50- and 80-week-old Polg ${ }^{D 181 A}$ mice (Fig. 4c) and further saw a significant difference between the identity scores of deletions $<100 \mathrm{bp}$ and $>100 \mathrm{bp}$ at 50 - and 80 -week-old Polg $^{D 181 A}$ as well as WT mice (Additional file 1: Fig. S2e). These data imply a differential contribution of non-direct repeat sequence similarity to short and long deletions.

\section{Abundant NCR multimers are exclusive to Polg ${ }^{D 181 A}$ _ expression}

As expected, the sequencing coverage exhibited some variability, likely associated with a slight sequence specificity of the transposase used for library preparation
[27-29]. However, in specific brain regions from the 50and 80-week-old Polg ${ }^{D 181 A}$ mice, we observed an increased coverage in the $15 \mathrm{~kb}+$ region including at least a part of the NCR (Additional file 1: Fig. S3a). Localised increased coverage is often thought to be associated with duplicated regions. As mitochondrial DNA is circular, it is not possible to distinguish small duplications from very long-range deletions (VLRDs) (Additional file 1: Fig. S3b). We therefore wondered if our data of mtDNA deletions could support the presence of multimers. By classifying VLRDs as deletions $>15 \mathrm{~kb}$, we found that VLRDs are specifically enriched in NAc and PVT from 50- and 80-week-old Polg ${ }^{D 181 A}$ mice (Fig. 5a) and enriched in the $15 \mathrm{~kb}+$ region (Fig. $5 \mathrm{~b}$ and Additional file 1: Fig. S3c), supporting the idea that mtDNA multimers including at least part of the NCR accumulate in a brain region-specific and $P \lg ^{D 181 A}$-dependent manner with
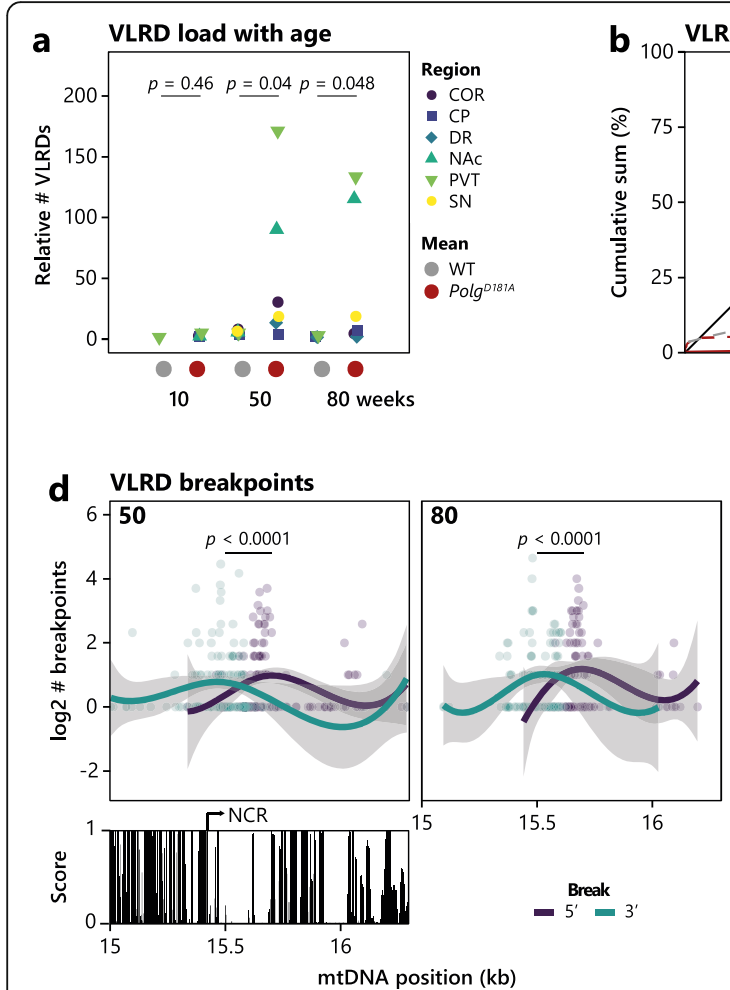
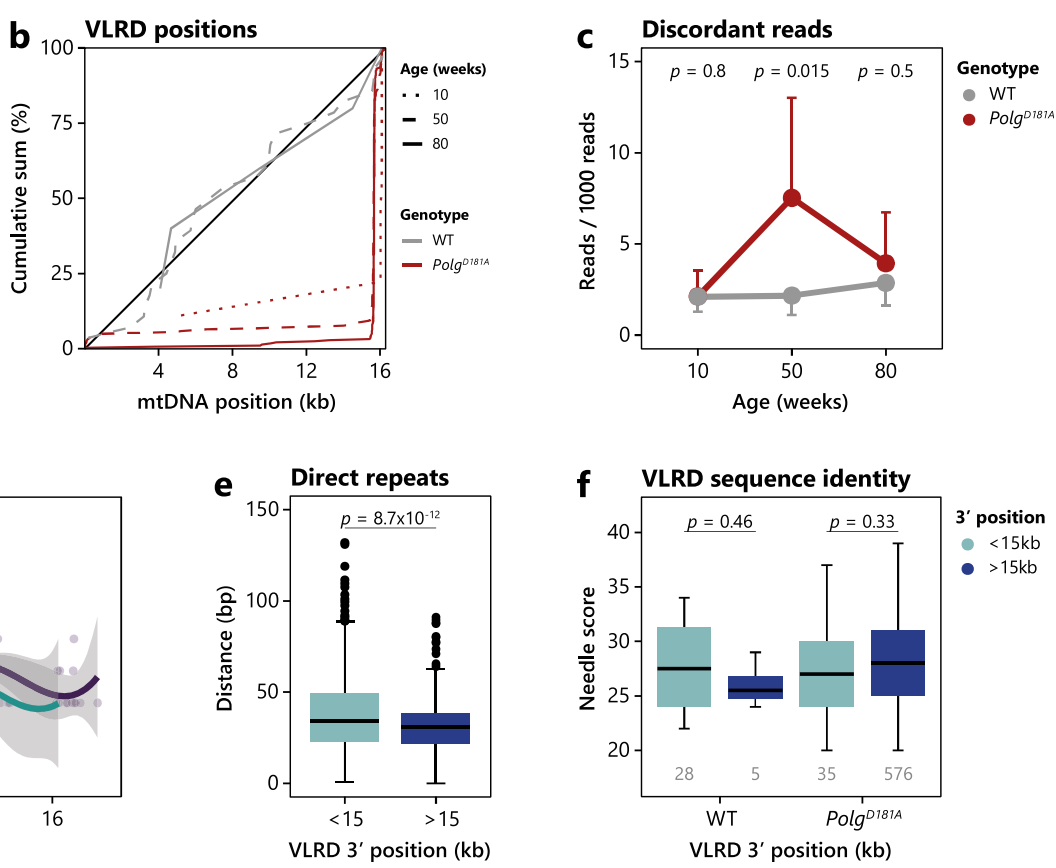

Fig. 5 Putative NCR multimers are Polg ${ }^{D 181 A}$-specific and accumulate with age in a highly brain region-specific manner. a Dot plot illustrating the age-dependent increase in the load of VLRDs in Polg ${ }^{D 181 A}$ mice across the investigated brain regions (as indicated by the colour legend). All samples have been normalised to the sample with the lowest number of detected variants. $\mathbf{b}$ Cumulative percentage of $5^{\prime}$ position of VLRDs (i.e. start position of the putative multimeric sequence) summed across brain regions for WT (grey) and Polg ${ }^{D 181 \mathrm{~A}}$ (red) for 10- (dotted line), 50(dashed line), and 80-week-old (full line) mice. c Mean number of discordant reads as extracted by samtools at 10, 50, and 80 weeks for WT (grey) and Polg ${ }^{D 181 A}$ (red) and standard deviation is indicated. $p$ values of two-sided $t$ tests are shown. $\mathbf{d}$ Summed analysis of mtDNA breakpoints of VLRDs from Polg ${ }^{181 A}$ mice in the NCR and surrounding region. 5' (purple) and 3' (dark turquoise) breakpoints are summed at each position across all brain regions at either 50 (left) or 80 (right) weeks old, and smooth conditional means are plotted. The lower panel shows the phastCons conservation score via the UCSC genome browser in the same region. $p$ values of two-sided $t$ tests are shown. e Boxplot showing the shortest average distance to a direct repeat of all Polg D181A VLRDs separated by VLRD $5^{\prime}$ position into $<15 \mathrm{~kb}$ (light blue) or $>15 \mathrm{~kb}$ (dark blue). $P$ values of two-sided $t$ tests are shown. $\mathbf{f}$ Boxplot showing the Needle identity score of WT and Polg ${ }^{\text {D181A }}$-derived VLRDs pooled across ages and brain regions examined split into VLRDs with $3^{\prime}$ position $<15 \mathrm{~kb}$ (light blue) and $>15 \mathrm{~kb}$ (dark blue). $p$ values of two-sided Wilcoxon tests are shown 
age. We also found an increase in discordant reads in 50-week-old Polg ${ }^{D 181 A}$ mice, which further supports the presence of genomic rearrangements such as multimers (Fig. 5c and Additional file 1: Fig. S3c).

These putative multimers appear to form in a quite restricted region of mtDNA as their $5^{\prime}$ and $3^{\prime}$ "breakpoints" (indicating the end and the start of the duplicated sequence, respectively), accumulate at rather discrete positions (Fig. 5d) spanning a region with a low conservation score across mammals (Fig. 5d, bottom panel). Previous data suggested the presence of multimers in the brain from the mutator mouse [16]. We used the same PCR approach as Williams et al. and validated the Polg $^{D 181 A}$-specific presence of multimers (Additional file 1: Fig. S4a, top and middle panel). An alternative PCR setup that would only yield a product in the presence of multimers confirmed these results (Additional file 1: Fig. S4a, bottom panel) and subsequent data also indicated the presence of inversions (Additional file 1: Figs. S4b,c).

Together, these data support the presence of highly brain region-specific ageing-induced Polg ${ }^{D 181 A}$ dependent multimers which are highly specific to a partial NCR-containing segment of mtDNA specifically in NAc and PVT.

\section{Direct repeats may be involved in NCR multimer formation}

Multimers can be formed by several mechanisms. One mechanism is by strand slipping during replication which may be influenced by the local environment surrounding the NCR, which is known to interact with the inner mitochondrial membrane [9]. Another mechanism is mediated by the DNA sequence surrounding the start and end positions of the multimer region. In support of the idea of strand slipping, we find VLRDs in the $15 \mathrm{~kb}+$ region to be closer to direct repeats compared to multimers in other parts of the mtDNA (Fig. 5e), though the overall sequence similarity surrounding breakpoints is not different (Fig. 5f). SNVs were enriched near VLRD $5^{\prime}$ breakpoints as well as $\sim 7 \mathrm{~kb}$ upstream with a mean distance of $75 \pm 462$ bp to the nearest SNV (Additional file 1: Fig. S5a). Fifteen percent of VLRD breakpoints co-position with SNVs, a number which is not influenced by discordant reads. SNVs were not enriched within the putative multimeric region (Additional file 1: Fig. S5b).

\section{Transgene expression level does not drive variants}

The expression of transgenes are often not similar across tissues, which is also true for Polg ${ }^{D 181 A}$ expression [14]. To confirm that the expression differences were not driving the differences we observed in the accumulation of mutations in response to Polg ${ }^{D 181 A}$ expression, we evaluated the expression levels of endogenous Polg and transgenic Polg ${ }^{D 181 A}$. Importantly, we were interested in the relative expression levels of the two transcripts, as endogenous and transgenic Polg will be competing for access to mtDNA during replication. As presented in previous sections, we find no correlation between mtDNA mutation levels and relative Polg ${ }^{\text {D181A }} /$ Polg levels at any age (Additional file 1: Fig. S5c). Together, this demonstrates that transgene expression levels were not the major driver of brain region specificity to proofreading deficiency in mitochondria.

\section{mtDNA variants cluster together along genomic regions}

Throughout our analysis of the mutation spectrum of mtDNA from both WT and Polg ${ }^{D 181 A}$ mice, it became increasingly clear that different types of variants often were found in specific mtDNA regions. To further investigate this, we plotted all variants analysed-SNVs, deletions, VLRDs (i.e. multimers)-across mtDNA in a circular plot (Fig. 6a). Visual inspection of this plot showed that different types of variants are enriched in the vicinity of each other. We found a strong, positive correlation between SNV and deletion load at the gene level which is independent of ageing and genotype (Fig. 6b), indicating positional sensitivity to the accumulation of mutations which may reveal underlying genomic instability in specific regions or be caused by higher order structures.

\section{Discussion}

Here we report a brain-wide spatio-temporal map of the mtDNA mutation spectrum in WT and proof-readingdeficient mice expressing Polg ${ }^{D 181 A}$ under the CaMKII $\alpha$ promoter. Using a PCR-free approach to enrich mtDNA for next-generation sequencing, we were able to study small tissue dissections while minimising bias to the analysis. Using this approach, we found that (1) the ageing-induced increase in SNVs and deletions is largely brain region-independent and reaches saturation at 50 weeks; (2) Polg ${ }^{D 181 A}$ expression specifically increase SNV and deletion levels in COR, NAc, and PVT; (3) ageing increases the number of shared SNVs, a feature that is enhanced in Polg ${ }^{D 181 A}$ mice and SNVs are prominent in the NCR; (4) deletions follow a bimodal size distribution independent of age and genotype; and (5) Polg ${ }^{D 181 A}$ induces NCR-containing multimers specifically in NAc and PVT in an ageing-dependent manner.

Deletions have been described in e.g. the brain [17, 30-34], muscle [35-37], and heart [30, 37]; however, all these studies suffer from limitations in either the amount of tissue required for input, a priori bias to the analysis, or limited temporal insight. Early studies tend to argue that deletions are mainly major arc deletions between the two origins of replication; however, we do 


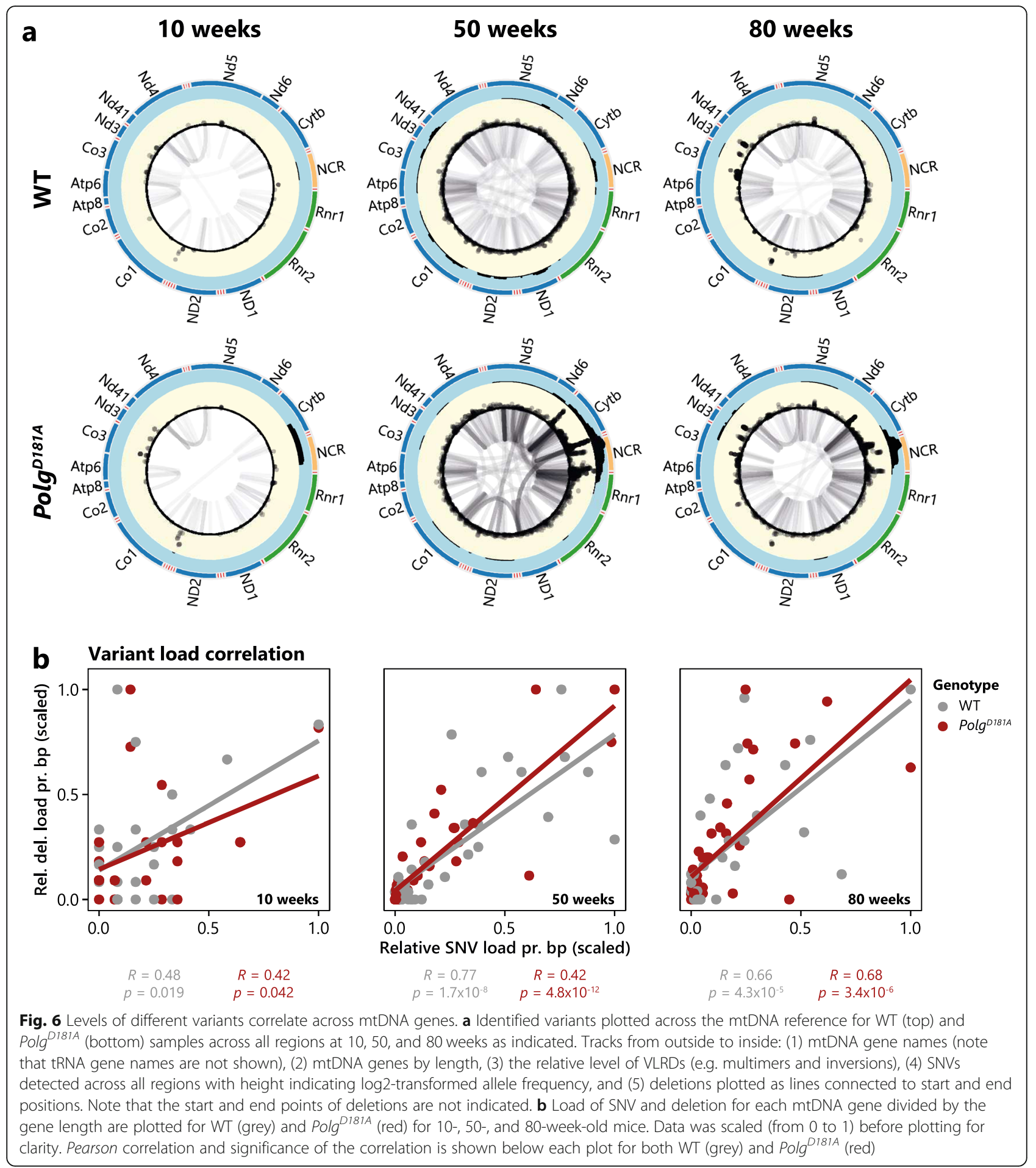

not find any indication of specific accumulation of common mtDNA deletion between the origins of replication (Figs. 3b and 4a), similar to the mutator mouse [16]. Instead, we identified a highly diverse deletion spectrum ranging from $1 \mathrm{bp}$ to $15 \mathrm{~kb}$ with a tendency for very short $(<10 \mathrm{bp})$ deletions to preferentially accumulate across all ages (Fig. 4b). The bimodal distribution of deletion lengths (Fig. 4b) was quite surprising. MtDNA deletions may be subject to two opposite working mechanisms: (1) shorter molecules, caused by larger deletions, finish replication faster, in principle leading to their rapid accumulation [23, 38]; (2) mtDNA undergo purifying selection [39], leading to the preferential loss of deleterious molecules (e.g. larger deletions) [40]. This 
bimodal distribution might be explained by the combination of these two mechanisms. However, the positive selection hypothesis has been disproved [41], and random genetic drift as the major contributor to the accumulation of short mtDNA species [42] was also recently questioned [43]. Furthermore, the presence of large deletions will naturally restrict the presence of smaller deletions. The description of a tight link between transcription and replication has increased interest in the positive selection idea [44], but the mechanisms remain unknown. However, the fact that young mice have a higher proportion of very small deletions (Fig. 4b) could favour a hypothesis including positive selection.

mtDNA deletions have been proposed to be prevalent between direct repeats $[45,46]$ in both normal ageing and disease, though this is not always the case $[47,48]$. As such, deletions are grouped into type I (flanked by direct repeat) and type II (not flanked by direct repeat) [49]. Type I deletions are hypothesised to be formed by polymerase slippage during replication [50]. The discrepancy between human disease cases [51] and mouse models regarding direct repeats may relate to the different involvement of direct repeats in deletion formation between long-lived and short-lived mammals [26]. Type II deletions colocalize with $2 \mathrm{D}$ and 3D mtDNA structures [49] and occur spontaneously during replication either through strand slipping [48], Polg stalling [52], or repair of double-stranded breaks [53]. The fact that Polg ${ }^{D 181 A}$ expression increases deletion load without affecting the distribution of deletion sizes remains elusive. We speculate that Polg ${ }^{D 181 A}$-induced replication instability would increase the frequency of deletion-mediating events without effecting their overall properties or characteristics, but increased SNVs in itself do not seem to increase deletion rate [54].

Studies on duplications in mtDNA have previously focused on the D-loop [55-57], and we similarly found duplications to be a brain region- and NCR-specific ageing-induced Polg ${ }^{D 181 A}$-dependent event (Fig. 5a). NCR duplications could be caused by Polg stalling during replication [58]. Data indicates that the D-loop may not be an ideal region to use as a control in the estimation of mtDNA copy number [59], which could be related to the propensity of duplications in this region and may be partly due to direct repeats (Fig. $5 \mathrm{~d}$ ). In addition, the D-loop is often chosen for primer design for longrange PCR, why such long-range PCR is not suitable for rearrangement analysis without a priori knowledge of the multimer landscape which may be highly dependent on the animal model, the disease progression, or the tissue investigated. The D-loop has been proposed to directly interact with the inner mitochondrial membrane and through this interaction mediates protein recruitment and mitochondrial structure [12]. Multimers of
NCR may therefore influence mitochondrial function in other ways than the classical view of energy production and mtDNA copy number.

We observed no differences in the types of SNV with ageing in neither WT nor Polg ${ }^{D 181 A}$ mice indicating that age-induced oxidative damage is not a major driver of mutations in mtDNA but rather mutations are caused by the accumulation of Polg errors in both WT and Polg ${ }^{D 181 A}$ mice. Oxidative damage of mtDNA causes 8oxo-dG which was argued to result in $\mathrm{G}>\mathrm{T}$ transversions [60]; however, we find that $\mathrm{G}>\mathrm{T}$ only take up a small fraction of the identified SNVs, which could indicate that Polg is able to correctly incorporate T opposite 8oxo-dG under oxidative conditions [61]. In support of this, oxidative damage is limited in mutator mice $[6,22$, 62], data which can likely be extrapolated to our model. Instead, $\mathrm{C}>\mathrm{T}$ and $\mathrm{T}>\mathrm{C}$ are the major identified SNVs (Additional file 1: Fig. S1e). The major base interpretation mistake by Polg is T-GTP mispairing [63-66], though $A>G$ can also occur due to deamination of adenosine. $\mathrm{C}>\mathrm{T}$ is generally associated with cytosine deamination, though the exact link to Polg function is not clear. Overall, the mutation spectrum identified here is similar to that of the mutator mouse [19] as well as mtDNA analysis of ChIP-seq-derived data [24].

We found that SNVs tend to cluster in hotspots which display genotype and ageing characteristics (Fig. 2d). We found another Polg ${ }^{D 181 A}$-specific mtDNA mutation trait, the presence of multimers (Fig. 5a). The presence of several Polg ${ }^{D 181 A}$-dependent mutation traits compared to WT should caution the use of mtDNA mutationinducing mouse models to describe ageing processes. We cannot testify to the tissue specificity of these observations, but at least the heart from mutator mice also seems to harbour genotype-specific multimers [16]. These mutations may not reflect the naturally occurring ageing phenotype adequately.

An unanswered question remains: what is the cause of regional sensitivity of proof-reading deficiency? As mitochondria are highly dynamic organelles that constantly undergo fission and fusion, the rates of these processes influence both replication and turnover of mtDNA. This is especially true in neurons, where mitochondria can roughly be divided into those found in the soma and those at the synapse with a constant transport of mitochondria along the axon $[67,68]$. In addition, Parkin has been shown to protect SN neurons from the accumulation of mtDNA mutations in the mutator mouse [69], and it is likely that Parkin or other proteins influence similar processes in different brain regions. As the total level of Polg also appears to influence the propagation of deleterious mtDNA molecules [70], the transgenic expression of Polg may influence this regulation. 
Elucidation of the cause and effect relationship between mtDNA mutations and ageing is not so straightforward. In mice, there is evidence that mtDNA SNVs themselves are adequate to induce premature ageing [71, 72], though the SNV load in these models is several fold higher than that observed in aged humans [73], and heterozygous Polg $^{D 257 A}$ mice that also have elevated SNV levels do not show signs of premature ageing [6]. Human data suggest the ageing-dependent accumulation of mtDNA deletions [74], but whether this is a cause or consequence of ageing is not clear. Interestingly, mice lacking Mgme1, the mitochondrial exonuclease, accumulate deletions but do not show premature ageing [75], indicating the requirement of SNVs, not deletions, for premature ageing, likely by affecting the functionality of mitochondrial proteins or functional RNAs.

In all, our data provide a novel view of the spatiotemporal accumulation of mtDNA mutations by providing a method for investigating the full mutation spectrum from very limited tissue dissections. The differential response across brain regions to a state of replication instability provides insight into a possible heterogenic mitochondrial landscape across the brain that may help explain the specificity of neuropsychiatric disorders in individuals with mitochondrial disease as well as neurological changes associated with ageing. Appreciating the tissue and region specificities of the mitochondrial genome in terms of copy number variations $[59,76]$, mutations [14], or gene expression [77] is pivotal to understand changes in mitochondrial dynamics in ageing and disease states.

\section{Conclusions}

We provide a novel unbiased spatio-temporal mapping of the full mtDNA mutation spectrum of discrete regionspecific dissections of mouse brain using an approach that does not require PCR amplification of mtDNA prior to library preparation. We demonstrate that both single nucleotide variants (SNVs) and deletions accumulate homogeneously across the examined brain regions during ageing from 10 to 50 weeks but see no further increase towards 80 weeks. In mice expressing proof-reading-deficient Polg, Polg ${ }^{D 181 A}$, the mitochondrial response to this state of replication instability is highly brain region-specific, and we demonstrate that the paraventricular thalamic nucleus and nucleus accumbens are mutational hotspots. The increased mutation load in ageing Polg ${ }^{D 181 A}$ mice compared to wildtype is only moderately associated with changes in mutation characteristics. Polg ${ }^{D 181 A}$ also induces an ageingdependent accumulation of non-coding control-region multimers, a feature that appears almost non-existent in wild-type mice. Our data show that unbiased sequencing of mtDNA from small tissue dissections can contribute to our understanding of the heterogenous mtDNA regulatory processes in ageing and disease states.

\section{Methods \\ Animals}

All animal care and experimental procedures were in accordance with the guidelines for proper conduct of animal experiments published by Science Council of Japan and approved by RIKEN Wako Animal Experiment Committee. All CaMKII $\alpha$-Polg ${ }^{D 181 A}$ transgenic mice used were heterozygotes. Animals were bred as described previously $[14,78]$. In brief, mutant male CaMKII $\alpha-$ Polg $^{D 181 A}$ mice were mated with wild-type (WT) C57BL/6J female mice. Genotyping was performed using genomic DNA isolated from tail biopsies as described [14].

\section{Tissue sampling}

Heterozygous female Polg mice (Tg (CaMKII $\alpha-$ Polg $\left.{ }^{\mathrm{D} 181 \mathrm{~A}}\right)$ C57BL/6J) and female WT littermates (1011 weeks (10-week age group), 48-49 weeks (50-week age group), $81-84$ weeks (80-week age group)) ( $n=4-6$ for each condition) were sacrificed by cervical dislocation, and the head was immediately removed and submerged in ice-cold modified ACSF (10 mM HEPES, 125 $\mathrm{mM} \mathrm{NaCl}, 5 \mathrm{mM} \mathrm{KCl}, 2 \mathrm{mM} \mathrm{CaCl}, 2 \mathrm{mM} \mathrm{MgSO}$, 10 $\mathrm{mM}$ glucose, $\mathrm{pH}$ 7.4). The whole brain was removed and washed in ice-cold ACSF and cut in 1-mm cortical sections using a brain matrix (ASI instruments, RBM2000C). Areas of interest were identified and immediately dissected in ice-cold ACSF and snap-frozen in liquid nitrogen.

\section{mtDNA enrichment}

Each sample was incubated at $37^{\circ} \mathrm{C}$ for $16 \mathrm{~h}$ in $90 \mu \mathrm{L}$ DNA buffer $(10 \mathrm{mM}$ TrisHCl pH 8.0, $0.1 \mathrm{M} \mathrm{NaCl}, 1 \%$ SDS) and $10 \mu \mathrm{L}$ Proteinase $\mathrm{K}$ (Roche, \#03115828001) and treated with RNase A for $10 \mathrm{~min}$ at room temperature. Total DNA was extracted using 1 vol AMPure XP beads (Beckman Coulter, \#A63881). A small aliquot of the purified DNA (1/10 vol) was stored for $\mathrm{qPCR}$ and the remainder used for mtDNA enrichment. Total DNA was exonuclease treated for $36 \mathrm{~h}$ at $37^{\circ} \mathrm{C}$ with interval shaking $(1000 \mathrm{rpm})(18 \mu \mathrm{L}$ total DNA, $3 \mu \mathrm{L}$ NEBuffer 4 (10x), $4 \mu \mathrm{L}$ ExoV (NEB, \#M0345L), $6 \mu \mathrm{L}$ ATP $(10 \mathrm{mM}))$. Additional $1 \mu \mathrm{L}$ NEBuffer $4(10 \mathrm{x}), 2 \mu \mathrm{L}$ ExoV, $6 \mu \mathrm{L}$ ATP $(10 \mathrm{mM})$ was added and incubated at $37^{\circ} \mathrm{C}$ for $16 \mathrm{~h}$. DNA was extracted using 0.4 vol AMPure XP beads. A small aliquot of the purified DNA (1/10 vol) was saved for qPCR. We note that the initial crude extraction of total DNA was unsuitable for the cerebellum due to the high lipid content of this tissue. For samples with high fibre density (e.g. NAc), we also periodically experienced some issues with solubility which was solved with either increased incubation time or increased buffer volume. 


\section{qPCR validation of ExoV treatment}

DNA stored before and after ExoV treatment was diluted and used to evaluate nDNA and mtDNA in the samples. qPCR conditions: 1 cycle $\left(95^{\circ} \mathrm{C} 30 \mathrm{~s}\right), 40$ cycles $\left(95^{\circ} \mathrm{C} 5 \mathrm{~s}, 60^{\circ} \mathrm{C} 30 \mathrm{~s}\right), 1$ cycle $\left(95^{\circ} \mathrm{C} 15 \mathrm{~s}, 60^{\circ} \mathrm{C} 60 \mathrm{~s}\right.$, $95^{\circ} \mathrm{C} 15 \mathrm{~s}$ ) (QuantStudio 12k Flex System, Applied Biosystems) using SYBR Premix Ex Taq (Takara, RR041) in $5-\mu \mathrm{L}$ reaction volume. Primer sequences can be found in Additional file 2: Table S1.

\section{Library preparation and sequencing}

Up to $0.5 \mathrm{ng}$ DNA was prepared for sequencing using the NexteraXT DNA kit (Illumina, \#FC-131-1024). Libraries were quantified using KAPA Universal kit (Kapabiosystems, \#UKK4824) or Qubit dsDNA HS Assay (Invitrogen, \#Q32851) and library size estimated by BioAnalyzer HS DNA chip (Agilent, \#5067-4626). Libraries were pooled to $2 \mathrm{nM}$ and sequenced on the Illumina MiSeq with 150-bp paired end reads (Illumina, \#MS-102-2002). Note that the same number of PCR cycles for library amplification was used independent of start DNA input and that this did not lead to low complexity libraries or affected library size distribution as evaluated by BioAnalyzer.

\section{Sequencing analysis}

Sequencing data was filtered, trimmed, mapped, and variant called using the BBTools suite (38.07) [21] using Clumpify (dedupe), FilterByTile (default), BBDuk (first: $\mathrm{ktrim}=\mathrm{r} \mathrm{k}=23$ mink $=11$ hdist $=1$ tbo tpe minlen $=100$ $\mathrm{ftm}=5$ ordered (using adaptor resource provided with BBTools); second: $\mathrm{k}=27$ ordered qtrim $=\mathrm{r}$ trimq $=8$ (using sequencing artefacts and phiX sequences provided with BBTools)), BBMap (vslow $\mathrm{k}=11$ secondary $=\mathrm{t}$ minratio $=0.55$ tipsearch $=300$ maxindel $=160000$ ambig $=$ all rescuedist $=30000$ qtrim $=1 \mathrm{r}$ ), and CallVariants (rarity $=0.005$ minallelefraction $=0.005$ ploidy $=100$ minedistmax $=5$ border $=5$ minquality $=10$ minqualitymax $=$ 10 minscore $=10$ ) and postfiltered in $\mathrm{R}$ for minimum supporting reads (SNVs 4, deletions 2), sequencing depth (SNVs 50, deletions 25), and quality score (SNVs 20 , deletions 10 ). Initially, reads were mapped to $\mathrm{mm} 10$ (ensembl) without the mitochondrial chromosome (MT) (using BBMap perfect mode) and unmapped reads were re-mapped to a modified version of MT consisting of two tandem MT sequences (in principle, a "double" MT chromosome, which we termed dMT) in order to call long-range deletions. We note that deletion calling is sensitive to kmer length chosen during mapping. Whereas a longer kmer showed more reproducible results, shorter kmers resulted in better detection sensitivity of deletions. Thus, we chose the shortest kmer value within the higher kmer lengths that gave reproducible results. The same number of aligned reads was used for each sample for variant analysis using random sampling with BBTools Reformat to avoid bias due to sequencing depth. Because of this approach, it is not necessary to normalise the read depth by the number of total reads. We filtered deletions to be a maximum of $15 \mathrm{~kb}$ based on our previous data indicating the presence of $\sim 2 \mathrm{~kb}$ mtDNA molecules in the Polg mice [13]. For the analysis of multimers, only deletions with a length $>15 \mathrm{~kb}$ were retained, so no variants were included in both analyses.

SNV effect was evaluated using SnpEff [79]. Multisample overlap and statistics (Additional file 1: Figure S1C) was calculated using SuperExactTest [80]. Discordant reads were extracted using samtools (-F 1294).

The in silico deletion length-matched libraries were generated by randomly sampling $n$ mtDNA positions (where $n$ is the number of deletions in either WT or Polg ${ }^{D 181 A}$ samples), and these positions were used as deletion start positions. Deletion lengths (of either WT or Polg ${ }^{D 181 A}$ deletion libraries) were randomly shuffled and assigned to each randomly sampled mtDNA position, and the sum of these denoted the end position of the in silico generated deletions.

Statistical tests used are indicated in individual figure legends and were performed in $R$ using base functions. To test the influence of ageing, genotype, animal, and/or region on the accumulation of mtDNA variants, we performed two- or three-way ANOVA. For pairwise testing, a two-sided $t$ test (Welch's) was performed. For pairwise testing of non-normal distributed data (as evaluated by Shapiro-Wilk's method) that does not fulfil the central limits theorem, Wilcoxon testing was performed. All $p$ values were Bonferroni corrected. Circular plots were made using the $\mathrm{R}$ package circlize [81].

To detect direct repeats in mtDNA, we split MT into 100-bp non-overlapping fragments and composed a local blast database (makeblastdb -in 100bp_fragments.fa -input_type fasta -dbtype nucl -out blastdb_100bp) against which we blasted the mtDNA sequence (blastn -task blastn-short -num_descriptions 500000000 num_alignments 500000000 -ungapped query mtDNA.fa -db blastdb_100bp -word_size 5 -evalue 1e300 -outfmt 6 out mtblast). Putative direct repeats were post filtered to include maximum 1 mismatch per 4 nucleotides and for a minimum size of $8 \mathrm{bp}$ due to the high frequency of shorter direct repeats.

Sequence identity scoring was performed using Needle (needleall in1.fa in2.fa -gapopen 10 -gapextend 0.5 -outfile out.txt) from EMBOSS [82].

\section{PCR and cloning}

Total DNA was prepared from two separate littermate pairs (female) 35-37 weeks old as described above. PCR was performed using Tks Gflex (Takara, \#R060A) with $5 \mathrm{ng}$ total DNA/reaction. PCR was run using 1 cycle: 
$94{ }^{\circ} \mathrm{C} 2 \mathrm{~min} ; 30$ cycles: $94{ }^{\circ} \mathrm{C} 15 \mathrm{~s}, \mathrm{X}^{\circ} \mathrm{C} 20 \mathrm{~s}, 68^{\circ} \mathrm{C}$ Ys; 1 cycle: $68^{\circ} \mathrm{C} 7 \mathrm{~min}$, where $\mathrm{X}$ and $\mathrm{Y}$ were optimised for each primer set used. PCR products were run on $1 \%$ agarose gels in TAE buffer, and DNA was visualised using ethidium bromide. Primer sequences and primerspecific PCR conditions can be found in Additional file 2: Table S2. PCR products were purified from $1 \%$ agarose gels using Wizard SV Gel and PCR Clean-Up System (Promega), cloned (Invitrogen, \#45-1641), and sequenced (BigDye Terminator v3.1, Applied Biosystems).

\section{RNA analysis}

Tissues from 4 pairs of female Polg mice and littermate controls (10-12 weeks) were collected as described above but were immediately stored in TRIzol after dissection instead of snap-freezing. RNA was purified using the Direct-zol RNA Microprep Kit (Zymo Research, \#R2060). One hundred nanograms of random hexamers (Invitrogen) was annealed $\left(85^{\circ} \mathrm{C}, 5 \mathrm{~min}\right)$ to $100 \mathrm{ng}$ RNA. Reverse transcription was carried out with 100 U M-MLV Reverse Transcriptase (Invitrogen, \#28025013) in a $13-\mu \mathrm{L}$ volume supplemented with 10 $\mathrm{mM}$ DTT and $1 \mathrm{mM}$ dNTP (NEB, \#N0447S) at room temperature, $10 \mathrm{~min}$, then at $37^{\circ} \mathrm{C}, 60 \mathrm{~min}$. cDNA was used for qPCR in technical triplicates. qPCR was performed as above. Primer sequences can be found in Additional file 2: Table S3.

\section{Supplementary information}

Supplementary information accompanies this paper at https://doi.org/10. 1186/s12915-020-00890-5.

\section{Additional file 1: Supplementary figures 1-5. Fig. S1: Additional} analysis of mtDNA single nucleotide variants. Fig. S2: Additional analysis of mtDNA deletions. Fig. S3: Additional analysis of mtDNA multimers. Fig. S4: Additional analysis of mtDNA multimers and inversions. Fig. S5: Polg expression analysis.

Additional file 2: Supplementary tables 1-3. Table S1: Table of primers used for qPCR and RT-qPCR to assess the levels of mtDNA, nDNA and mRNA targets. Table S2: Table of primers used for PCR to assess structural variants of mtDNA. Table S3: Table of primers used for qPCR to assess the expression of endogenous and transgenic Polg ${ }^{\text {D181A }}$.

\section{Acknowledgements}

We thank the Research Resource Division, RIKEN Center for Brain Science, for the technical assistance.

\section{Authors' contributions}

EKB performed most of the experiments and performed all analyses. Dissection of small brain regions was performed by EKB and NF-T and supervised by MK-S. T. Kasahara contributed to the data interpretation. EKB and T. Kato conceived the study and wrote the paper. The authors read and approved the final manuscript.

\section{Funding}

This work was supported by Japan Society for the Promotion of Science KAKENHI (18K15531 to EKB, 18H05435 and 17H01573 to T.Kato) and the Advanced Genome Research and Bioinformatics Study to Facilitate Medical Innovation (GRIFIN) from the Japan Agency for Medical Research and Development (AMED) (19km0405208h0004 to T.Kato).

\section{Availability of data and materials}

Sequencing data has been deposited to the Sequencing Read Archive at NCBI under accession number PRJNA623951. Analytical tools used in this study are cited within the "Methods" section.

Ethics approval and consent to participate

All animal care and experimental procedures were in accordance with the guidelines for proper conduct of animal experiments published by Science Council of Japan and approved by RIKEN Wako Animal Experiment Committee.

\section{Consent for publication}

Not applicable.

\section{Competing interests}

The authors declare that they have no competing interests.

\section{Author details}

'Laboratory for Molecular Dynamics of Mental Disorders, RIKEN Center for Brain Science, Wako, Saitama, Japan. ${ }^{2}$ Current address: Support Unit for Bio-Material Analysis, Research Resources Division, RIKEN Center for Brain Science, Wako, Saitama, Japan. ${ }^{3}$ Current address: Career Development Program, RIKEN Center for Brain Science, Wako, Saitama, Japan. ${ }^{4}$ Department of Psychiatry and Behavioral Science, Juntendo University, Graduate School of Medicine, Hongo 2-1-1, Bunkyo, Tokyo 113-8421, Japan.

Received: 11 May 2020 Accepted: 6 October 2020

Published online: 23 October 2020

\section{References}

1. Alexeyev MF. Is there more to aging than mitochondrial DNA and reactive oxygen species? FEBS J. 2009;276:5768-87.

2. Kauppila JH, Stewart JB. Mitochondrial DNA: radically free of free-radical driven mutations. Biochim Biophys Acta. 2015;1847:1354-61.

3. Kauppila TES, Kauppila JHK, Larsson NG. Mammalian mitochondria and aging: an update. Cell Metab. 2017;25:57-71.

4. Roberts $L J$ 2nd, Reckelhoff JF. Measurement of F (2)-isoprostanes unveils profound oxidative stress in aged rats. Biochem Biophys Res Commun. 2001;287:254-6.

5. Hamilton ML, Van Remmen H, Drake JA, Yang H, Guo ZM, Kewitt K, Walter CA, Richardson A. Does oxidative damage to DNA increase with age? Proc Natl Acad Sci U S A. 2001;98:10469-74.

6. Trifunovic A, Wredenberg A, Falkenberg M, Spelbrink JN, Rovio AT, Bruder CE, Bohlooly YM, Gidlof S, Oldfors A, Wibom R, et al. Premature ageing in mice expressing defective mitochondrial DNA polymerase. Nature. 2004;429: 417-23.

7. Koopman WJ, Verkaart S, van Emst-de Vries SE, Grefte S, Smeitink JA, Nijtmans LG, Willems PH. Mitigation of NADH: ubiquinone oxidoreductase deficiency by chronic Trolox treatment. Biochim Biophys Acta. 2008;1777: 853-9.

8. Menzies KJ, Robinson BH, Hood DA. Effect of thyroid hormone on mitochondrial properties and oxidative stress in cells from patients with mtDNA defects. Am J Physiol Cell Physiol. 2009;296:C355-62.

9. Gerhold JM, Cansiz-Arda S, Lohmus M, Engberg O, Reyes A, van Rennes H, Sanz A, Holt IJ, Cooper HM, Spelbrink JN. Human mitochondrial DNAprotein complexes attach to a cholesterol-rich membrane structure. Sci Rep. 2015;5:15292.

10. Cuppari A, Fernandez-Millan P, Battistini F, Tarres-Sole A, Lyonnais S, Iruela G, Ruiz-Lopez E, Enciso Y, Rubio-Cosials A, Prohens R, et al. DNA specificities modulate the binding of human transcription factor $A$ to mitochondrial DNA control region. Nucleic Acids Res. 2019;47:6519-37.

11. Farge G, Falkenberg M. Organization of DNA in Mammalian Mitochondria. Int J Mol Sci. 2019;20(11):2770.

12. Holt IJ. The mitochondrial R-loop. Nucleic Acids Res. 2019;47:5480-9.

13. Kasahara T, Kubota M, Miyauchi T, Noda Y, Mouri A, Nabeshima T, Kato T. Mice with neuron-specific accumulation of mitochondrial DNA mutations show mood disorder-like phenotypes. Mol Psychiatry. 2006;11:577-93 523.

14. Kasahara T, Takata A, Kato TM, Kubota-Sakashita M, Sawada T, Kakita A Mizukami H, Kaneda D, Ozawa K, Kato T. Depression-like episodes in mice harboring mtDNA deletions in paraventricular thalamus. Mol Psychiatry. 2016;21:39-48. 
15. Kubota M, Kasahara T, Nakamura T, Ishiwata M, Miyauchi T, Kato T. Abnormal Ca2+ dynamics in transgenic mice with neuron-specific mitochondrial DNA defects. J Neurosci. 2006;26:12314-24.

16. Williams SL, Huang J, Edwards YJK, Ulloa RH, Dillon LM, Prolla TA, Vance JM, Moraes $C T$, Züchner $S$. The mtDNA mutation spectrum of the progeroid Polg mutator mouse includes abundant control region multimers. Cell Metab. 2010;12:675-82

17. Nido GS, Dolle C, Flones I, Tuppen HA, Alves G, Tysnes OB, Haugarvoll K, Tzoulis C. Ultradeep mapping of neuronal mitochondrial deletions in Parkinson's disease. Neurobiol Aging. 2017;63:120-7.

18. Ma H, Lee Y, Hayama T, Van Dyken C, Marti-Gutierrez N, Li Y, Ahmed R, Koski A, Kang E, Darby H, et al. Germline and somatic mtDNA mutations in mouse aging. PLoS One. 2018;13:e0201304.

19. Ni T, Wei G, Shen T, Han M, Lian Y, Fu H, Luo Y, Yang Y, Liu J, Wakabayash $Y$, et al. MitoRCA-seq reveals unbalanced cytocine to thymine transition in Polg mutant mice. Sci Rep. 2015;5:12049.

20. Jayaprakash AD, Benson EK, Gone S, Liang R, Shim J, Lambertini L, Toloue MM, Wigler M, Aaronson SA, Sachidanandam R. Stable heteroplasmy at the single-cell level is facilitated by intercellular exchange of mtDNA. Nucleic Acids Res. 2015;43:2177-87.

21. Bushnell B. BBMap short-read aligner, and other bioinformatics tools; 2015.

22. Kujoth GC, Hiona A, Pugh TD, Someya S, Panzer K, Wohlgemuth SE, Hofer T, Seo AY, Sullivan R, Jobling WA, et al. Mitochondrial DNA mutations, oxidative stress, and apoptosis in mammalian aging. Science. 2005:309:481-4.

23. Fuke S, Kametani M, Yamada K, Kasahara T, Kubota-Sakashita M, Kujoth GC, Prolla TA, Hitoshi S, Kato T. Heterozygous Polg mutation causes motor dysfunction due to mtDNA deletions. Ann Clin Transl Neurol. 2014;1:909-20.

24. Rensch T, Villar D, Horvath J, Odom DT, Flicek P. Mitochondrial heteroplasmy in vertebrates using ChIP-sequencing data. Genome Biol. 2016;17:139.

25. Samuels DC, Schon EA, Chinnery PF. Two direct repeats cause most human mtDNA deletions. Trends Genet. 2004;20:393-8.

26. Lakshmanan LN, Gruber J, Halliwell B, Gunawan R. Role of direct repeat and stem-loop motifs in mtDNA deletions: cause or coincidence? PLoS One. 2012;7:e35271.

27. Marine R, Polson SW, Ravel J, Hatfull G, Russell D, Sullivan M, Syed F, Dumas $\mathrm{M}$, Wommack KE. Evaluation of a transposase protocol for rapid generation of shotgun high-throughput sequencing libraries from nanogram quantities of DNA. Appl Environ Microbiol. 2011;77:8071-9.

28. Kia A, Gloeckner C, Osothprarop T, Gormley N, Bomati E, Stephenson M, Goryshin I, He MM. Improved genome sequencing using an engineered transposase. BMC Biotechnol. 2017;17:6.

29. Sato MP, Ogura Y, Nakamura K, Nishida R, Gotoh Y, Hayashi M, Hisatsune J, Sugai M, Takehiko I, Hayashi T. Comparison of the sequencing bias of currently available library preparation kits for Illumina sequencing of bacterial genomes and metagenomes. DNA Res. 2019;26:391-8.

30. Cortopassi GA, Shibata D, Soong NW, Arnheim N. A pattern of accumulation of a somatic deletion of mitochondrial DNA in aging human tissues. Proc Natl Acad Sci U S A. 1992;89:7370-4.

31. Bender A, Krishnan KJ, Morris CM, Taylor GA, Reeve AK, Perry RH, Jaros E, Hersheson JS, Betts J, Klopstock T, et al. High levels of mitochondrial DNA deletions in substantia nigra neurons in aging and Parkinson disease. Nat Genet. 2006;38:515-7.

32. Kraytsberg Y, Kudryavtseva E, McKee AC, Geula C, Kowall NW, Khrapko K. Mitochondrial DNA deletions are abundant and cause functional impairment in aged human substantia nigra neurons. Nat Genet. 2006;38:518-20.

33. Reeve AK, Krishnan KJ, Elson JL, Morris CM, Bender A, Lightowlers RN, Turnbull DM. Nature of mitochondrial DNA deletions in substantia nigra neurons. Am J Hum Genet. 2008;82:228-35.

34. Dolle C, Flones I, Nido GS, Miletic H, Osuagwu N, Kristoffersen S, Lilleng PK, Larsen JP, Tysnes OB, Haugarvoll K, et al. Defective mitochondrial DNA homeostasis in the substantia nigra in Parkinson disease. Nat Commun. 2016;7:13548.

35. Bua E, Johnson J, Herbst A, Delong B, McKenzie D, Salamat S, Aiken JM. Mitochondrial DNA-deletion mutations accumulate intracellularly to detrimental levels in aged human skeletal muscle fibers. Am J Hum Genet 2006;79:469-80.

36. Herbst A, Pak JW, McKenzie D, Bua E, Bassiouni M, Aiken JM. Accumulation of mitochondrial DNA deletion mutations in aged muscle fibers: evidence for a causal role in muscle fiber loss. J Gerontol A Biol Sci Med Sci. 2007;62: 235-45.

37. Simonetti S, Chen X, DiMauro S, Schon EA. Accumulation of deletions in human mitochondrial DNA during normal aging: analysis by quantitative PCR. Biochim Biophys Acta. 1992;1180:113-22.

38. Nekhaeva E, Bodyak ND, Kraytsberg Y, McGrath SB, Van Orsouw NJ Pluzhnikov A, Wei JY, Vijg J, Khrapko K. Clonally expanded mtDNA point mutations are abundant in individual cells of human tissues. Proc Natl Acad Sci U S A. 2002;99:5521-6.

39. Burr SP, Pezet M, Chinnery PF. Mitochondrial DNA Heteroplasmy and Purifying Selection in the Mammalian Female Germ Line. Dev Growth Differ 2018;60(1):21-32.

40. Ye K, Lu J, Ma F, Keinan A, Gu Z. Extensive pathogenicity of mitochondrial heteroplasmy in healthy human individuals. Proc Natl Acad Sci U S A. 2014; 111:10654-9.

41. Campbell G, Krishnan KJ, Deschauer M, Taylor RW, Turnbull DM. Dissecting the mechanisms underlying the accumulation of mitochondrial DNA deletions in human skeletal muscle. Hum Mol Genet. 2014;23:4612-20.

42. Elson JL, Samuels DC, Turnbull DM, Chinnery PF. Random intracellular drift explains the clonal expansion of mitochondrial DNA mutations with age. Am J Hum Genet. 2001;68:802-6.

43. Kowald A, Kirkwood TB. Mitochondrial mutations and aging: random drift is insufficient to explain the accumulation of mitochondrial deletion mutants in short-lived animals. Aging Cell. 2013;12:728-31.

44. Kowald A, Kirkwood TB. Transcription could be the key to the selection advantage of mitochondrial deletion mutants in aging. Proc Natl Acad Sci U S A. 2014;111:2972-7.

45. Madsen CS, Ghivizzani SC, Hauswirth WW. In vivo and in vitro evidence for slipped mispairing in mammalian mitochondria. Proc Natl Acad Sci U S A. 1993;90:7671-5.

46. Larsson NG. Somatic mitochondrial DNA mutations in mammalian aging. Annu Rev Biochem. 2010;79:683-706.

47. Srivastava S, Moraes CT. Double-strand breaks of mouse muscle mtDNA promote large deletions similar to multiple mtDNA deletions in humans. Hum Mol Genet. 2005;14:893-902.

48. Fukui $\mathrm{H}$, Moraes $\mathrm{CT}$. Mechanisms of formation and accumulation of mitochondrial DNA deletions in aging neurons. Hum Mol Genet. 2009;18: 1028-36.

49. Szczepanowska K, Trifunovic A. Origins of mtDNA mutations in ageing. Essays Biochem. 2017;61:325-37.

50. Shoffner JM, Lott MT, Voljavec AS, Soueidan SA, Costigan DA, Wallace DC. Spontaneous Kearns-Sayre/chronic external ophthalmoplegia plus syndrome associated with a mitochondrial DNA deletion: a slip-replication model and metabolic therapy. Proc Natl Acad Sci U S A. 1989;86:7952-6.

51. Hjelm BE, Rollins B, Morgan L, Sequeira A, Mamdani F, Pereira F, Damas J, Webb MG, Weber MD, Schatzberg AF, et al. Splice-Break: exploiting an RNA-seq splice junction algorithm to discover mitochondrial DNA deletion breakpoints and analyses of psychiatric disorders. Nucleic Acids Res. 2019:47:e59.

52. Damas J, Samuels DC, Carneiro J, Amorim A, Pereira F: Mitochondrial DNA rearrangements in health and disease-a comprehensive study 2014, 35:1-14.

53. Krishnan KJ, Reeve AK, Samuels DC, Chinnery PF, Blackwood JK, Taylor RW Wanrooij S, Spelbrink JN, Lightowlers RN, Turnbull DM. What causes mitochondrial DNA deletions in human cells? Nat Genet. 2008;40:275-9.

54. Wanrooij S, Luoma P, van Goethem G, van Broeckhoven C, Suomalainen A, Spelbrink JN. Twinkle and POLG defects enhance age-dependent accumulation of mutations in the control region of mtDNA. Nucleic Acids Res. 2004;32:3053-64.

55. Tengan $\mathrm{CH}$, Ferreiro-Barros $\mathrm{C}$, Cardeal M, Fireman MAT, Oliveira ASB, Kiyomoto BH, Gabbai AA. Frequency of duplications in the D-loop in patients with mitochondrial DNA deletions. Biochim Biophys Acta. 2002; 1588:65-70.

56. Bouzidi MF, Poyau A, Godinot C. Co-existence of high levels of a cytochrome b mutation and of a tandem 200 bp duplication in the D-loop of muscle human mitochondrial DNA. Hum Mol Genet. 1998;7:385-91.

57. Lee H-C, Pang C-Y, Hsu H-S, Weia Y-H. Ageing-associated tandem duplications in the D-loop of mitochondrial DNA of human muscle. FEBS Lett. 1994;354:79-83.

58. Bailey L, Cluett TJ, Reyes A, Prolla TA, Poulton J, Leeuwenburgh C, Holt IJ. Mice expressing an error-prone DNA polymerase in mitochondria display 
elevated replication pausing and chromosomal breakage at fragile sites of mitochondrial DNA. Nucleic Acids Res. 2009;37:2327-35.

59. Li B, Kaushik S, Kalinowski P, Kim B, Gershome C, Ching J, Poburko D. Droplet digital PCR shows the D-Loop to be an error prone locus for mitochondrial DNA copy number determination. Sci Rep. 2018:8:11392.

60. Brieba LG, Eichman BF, Kokoska RJ, Doublie S, Kunkel TA, Ellenberger T. Structural basis for the dual coding potential of 8-oxoguanosine by a highfidelity DNA polymerase. EMBO J. 2004;23:3452-61.

61. Graziewicz MA, Bienstock RJ, Copeland WC. The DNA polymerase gamma Y955C disease variant associated with PEO and parkinsonism mediates the incorporation and translesion synthesis opposite 7,8-dihydro-8-oxo-2'deoxyguanosine. Hum Mol Genet. 2007;16:2729-39.

62. Hiona A, Sanz A, Kujoth GC, Pamplona R, Seo AY, Hofer T, Someya S, Miyakawa T, Nakayama C, Samhan-Arias AK, et al. Mitochondrial DNA mutations induce mitochondrial dysfunction, apoptosis and sarcopenia in skeletal muscle of mitochondrial DNA mutator mice. PLoS One. 2010;5:e1 1468.

63. Nordmann PL, Makris JC, Reznikoff WS. Inosine induced mutations. Mol Gen Genet. 1988;214:62-7.

64. Longley MJ, Nguyen D, Kunkel TA, Copeland WC. The fidelity of human DNA polymerase gamma with and without exonucleolytic proofreading and the p55 accessory subunit. J Biol Chem. 2001;276:38555-62.

65. Song S, Pursell ZF, Copeland WC, Longley MJ, Kunkel TA, Mathews CK. DNA precursor asymmetries in mammalian tissue mitochondria and possible contribution to mutagenesis through reduced replication fidelity. Proc Natl Acad Sci U S A. 2005;102:4990-5.

66. Zheng W, Khrapko K, Coller HA, Thilly WG, Copeland WC. Origins of human mitochondrial point mutations as DNA polymerase gamma-mediated errors. Mutat Res. 2006;599:11-20.

67. Sheng ZH. The interplay of axonal energy homeostasis and mitochondrial trafficking and anchoring. Trends Cell Biol. 2017;27:403-16.

68. MacAskill AF, Kittler JT. Control of mitochondrial transport and localization in neurons. Trends Cell Biol. 2010;20:102-12.

69. Pickrell AM, Huang CH, Kennedy SR, Ordureau A, Sideris DP, Hoekstra JG, Harper JW, Youle RJ. Endogenous Parkin preserves dopaminergic substantia nigral neurons following mitochondrial DNA mutagenic stress. Neuron. 2015:87:371-81.

70. Chiang AC, McCartney E, O'Farrell PH, Ma H. A genome-wide screen reveals that reducing mitochondrial DNA polymerase can promote elimination of deleterious mitochondrial mutations. Curr Biol. 2019;29:4330-6 e4333.

71. Ross JM, Stewart JB, Hagstrom E, Brene S, Mourier A, Coppotelli G, Freyer C, Lagouge M, Hoffer BJ, Olson L, Larsson NG. Germline mitochondrial DNA mutations aggravate ageing and can impair brain development. Nature. 2013:501:412-5.

72. Ross JM, Coppotelli G, Hoffer BJ, Olson L. Maternally transmitted mitochondrial DNA mutations can reduce lifespan. Sci Rep. 2014;4:6569.

73. Khrapko K, Kraytsberg Y, de Grey AD, Vijg J, Schon EA. Does premature aging of the mtDNA mutator mouse prove that mtDNA mutations are involved in natural aging? Aging Cell. 2006;5:279-82.

74. Khrapko K, Bodyak N, Thilly WG, van Orsouw NJ, Zhang X, Coller HA, Perls TT, Upton M, Vijg J, Wei JY. Cell-by-cell scanning of whole mitochondrial genomes in aged human heart reveals a significant fraction of myocytes with clonally expanded deletions. Nucleic Acids Res. 1999;27:2434-41.

75. Matic S, Jiang M, Nicholls TJ, Uhler JP, Dirksen-Schwanenland C, Polosa PL, Simard ML, Li X, Atanassov I, Rackham O, et al. Mice lacking the mitochondrial exonuclease MGME1 accumulate mtDNA deletions without developing progeria. Nat Commun. 2018;9:1202.

76. Fuke S, Kubota-Sakashita M, Kasahara T, Shigeyoshi Y, Kato T. Regional variation in mitochondrial DNA copy number in mouse brain. Biochim Biophys Acta. 2011;1807:270-4.

77. Herbers E, Kekalainen NJ, Hangas A, Pohjoismaki JL, Goffart S. Tissue specific differences in mitochondrial DNA maintenance and expression. Mitochondrion. 2019:44:85-92.

78. Kato TM, Kubota-Sakashita M, Fujimori-Tonou N, et al. Ant1 mutant mice bridge the mitochondrial and serotonergic dysfunctions in bipolar disorder. Mol Psychiatry. 2018;23(10):2039-2049.

79. Cingolani P, Platts A, Wang le L, Coon M, Nguyen T, Wang L, Land SJ, Lu X, Ruden DM: A program for annotating and predicting the effects of single nucleotide polymorphisms, SnpEff: SNPs in the genome of Drosophila melanogaster strain $w^{1118}$; iso-2; iso-3. Fly (Austin) 2012, 6:80-92.

80. Wang M, Zhao Y, Zhang B. Efficient test and visualization of multi-set intersections. Sci Rep. 2015;5:16923.
81. Gu Z, Gu L, Eils R, Schlesner M, Brors B: circlize implements and enhances circular visualization in R. Bioinformatics 2014, 30:2811-2812.

82. Rice P, Longden I, Bleasby A. EMBOSS: the European molecular biology open software suite. Trends Genet. 2000;16:276-7.

\section{Publisher's Note}

Springer Nature remains neutral with regard to jurisdictional claims in published maps and institutional affiliations.

\section{Ready to submit your research? Choose BMC and benefit from:}

- fast, convenient online submission

- thorough peer review by experienced researchers in your field

- rapid publication on acceptance

- support for research data, including large and complex data types

- gold Open Access which fosters wider collaboration and increased citations

- maximum visibility for your research: over $100 \mathrm{M}$ website views per year

At BMC, research is always in progress.

Learn more biomedcentral.com/submissions 